\title{
Very light jets
}

\section{Axisymmetric parameter study and analytic approximation}

\author{
M. Krause` \\ Landessternwarte Königstuhl, 69117 Heidelberg, Germany
}

Received 7 May 2002 / Accepted 8 November 2002

\begin{abstract}
The propagation of extragalactic jets is studied by a series of twelve axisymmetric hydrodynamic simulations. Motivated by observational constraints, but unlike most previous simulations, the regime of jet to external medium density $(\eta)$ from $10^{-5}$ to $10^{-2}$ is explored, for Mach numbers $(M)$ between 2.6 and 26. The computational domain contained the bow shocks for the whole simulation time. The bow shocks are found to be spherical at source sizes below a critical value $r_{1}$ (blastwave phase), which can reach up to 10 jet radii. After that, their aspect ratio rises slowly, as long as the bow shock stays supersonic. The cocoons expand typically to almost the same size as the bow shock, unless the Mach number is below approximately three. Low values for the aspect ratio and the cocoon-to-bow-shock width ratio is demanded by recent Chandra X-ray observations of the bow shock in the archetypical radio galaxy Cygnus A. Therefore, $\eta<10^{-3}$ and $M<6$, in this source. The numerical work is complemented by an analytic approach for the spherical phase. Extending previous work, the radial force balance could be integrated for arbitrary background density and energy input, which results in a global solution. The analytic results are shown to be consistent with the numerical work, and a lower limit to $r_{1}$ can be calculated, which falls below the numerical results by a few jet radii. It is shown explicitely how a King density distribution changes the discussed aspects of the bow shock propagation. Because the jet head propagates very fast in the blastwave phase, it turns out that it is not possible to "frustrate" a jet by a high density environment. This is very important for the class of small radio galaxies (compact symmetric objects / $\mathrm{GHz}$ peaked sources): They have to be young. During its blastwave phase, a powerful jet can transfer typically $10^{60}$ erg to the environmental gas. This is enough to balance the radiative losses in a cooling flow, if one of the cluster galaxies harbours a powerful jet every $10^{9}$ years.
\end{abstract}

Key words. hydrodynamics - instabilities - shock waves - galaxies: jets

\section{Introduction}

Extragalactic jets have been known in the optical since the famous observation of the jet beam in M 87 (Curtis 1918). The understanding of those collimated flows improved considerably with the advent of radio astronomy (e.g. Baade \& Minkowski 1954). During that era, plenty of radio jets have been found in extragalactic objects. Surrounding the beams, a large cocoon with lower surface brightness was found, which can extend up to tens of jet radii (e.g. in Cygnus A Carilli \& Barthel 1996). They have been imaged down to the very center of the mainly elliptical host galaxies, where a supermassive black hole is believed to power the bipolar, relativistic, outflows by an accretion process. Jets can propagate up to several Mpc (Schoenmakers et al. 2001). According to standard jet theory, they displace the medium they propagate into and form a bow shock around the system. Although, the presence of bow shocks could be anticipated from some ROSAT images (Carilli et al. 1994), the Chandra X-ray observatory provided the first

\footnotetext{
* e-mail: M.Krause@lsw.uni-heidelberg.de
}

clear view on those features with satisfactory resolution (Smith et al. 2002). The bow shock in Cygnus A is a very fine example. The source is believed to be located nearly perpendicular to the line of sight (Krichbaum et al. 1998). Therefore, one can easily measure the true aspect ratio (bow shock diameter in jet direction over perpendicular bow shock diameter) from the Chandra image (Smith et al. 2002), which turns out to be 1.2. Another parameter that can be easily measured with the new Chandra data is the relative extention of radio cocoon and bow shock. Defining this ratio to be taken perpendicular to the jet axis through the core renders it independent from the viewing angle. One can in principle relate these observables to jet parameters via numerical simulation results. However, this is only possible if the bow shock stays within the computational domain during the simulation. Up to now only a few hydrodynamical simulations in the literature meet this requirement. Two examples can be found in Reynolds et al. (2002) and Krause (2002). In the latter case, a jet with a density contrast (jet over surrounding medium) $\eta=10^{-4}$ was simulated. This resulted in an aspect ratio of slightly more than unity, similar to Cygnus A. 
Jet simulations have been performed so far mainly with $10^{-2}<\eta<10$ (e.g. Norman et al. 1982, 1983; Clarke et al. 1986; Kössl \& Müller 1988; Lind et al. 1989; Cioffi \& Blondin 1992; Clarke 1993; Massaglia et al. 1996; Komissarov 1999; Tregillis et al. 2001; Krause \& Camenzind 2001). Those studies have been reviewed by Norman (1993) and Ferrari (1998). One main result was that light jets, i.e. less dense than the surrounding medium, develop extensive cocoons, and are therefore believed to be present in radio galaxies and quasars. The regime below that density, which will be called very light, was touched only occasionally.

Recently, parameter studies of jets were carried out by Carvalho \& O'Dea (2002a,b) and Saxton et al. (2002). These studies partly employ an open boundary condition on the jet injection side. In that respect, they are complementary to the study presented here. The problem with the open boundary condition is that the backflow can transport large amounts of energy off the grid. This is a problem in the case of very light jets (especially for young bipolar sources), since the backflow is very fast compared to the head propagation. A similar problem arises when the bow shock leaves the grid sideways. Saxton et al. (2002) have presented simulations with a closed injection side boundary and a big enough grid for the bow shock to remain inside for most of the simulation time.

In this paper a systematic study of very light jets with $\eta \in\left[10^{-5} ; 10^{-2}\right]$ and internal Mach number $M_{\mathrm{j}} \in[2.6 ; 26]$ is presented. The injection side boundary is closed, and the grid is big enough to follow the bow shock for the whole simulation time. Contrary to previous studies, the focus in this paper is on the jet physics specific for very low jet densities. First, observational constraints on the jet parameters are reviewed. It follows a description of the simulations. Afterwards, an analytic approximation for the bow shock propagation is given, and finally the results are discussed. The time unit used in the following is the Myr, an abbreviation for $10^{6}$ years.

\section{Constraints from observation}

In nearby radio galaxies, the movement of individual jet features can be observed in the inner 100 parsecs (e.g. Britzen et al. 2000). These measurements indicate apparent superluminal motion, which is a unique feature of relativistic velocities, very close to the speed of light $c$. The velocity at kpc scale is not so easy measurable. Carilli \& Barthel (1996) cite a value of $v_{\mathrm{J}} \approx 0.4 c$ for the jet velocity in the kpc scale jet of Cygnus A, estimated from the hot spot spectra and consistent with luminosity, minimum energy hotspot pressure, lack of internal Faraday dispersion in the lobes, and the jet-to-counterjet surface brightness ratio. However, it should be emphasised, that this number is quite uncertain.

Parma et al. (1999) measure the radio source lifetime by synchrotron aging. Their straight forward analysis yields a lifetime $t_{\mathrm{s}}<100 \mathrm{Myr}$ for low power sources and $t_{\mathrm{s}}<10 \mathrm{Myr}$ for high power ones. However, they point out that this discrepancy does not have to be real, since the estimate depends on the magnetic field, which is more uncertain in high power radio galaxies. Reducing the magnetic field to one fourth of the applied equipartition value pushes the high power sources to
$100 \mathrm{Myr}$, also. This would be supported by jet-to-counterjet length asymmetry constraints (Scheuer 1995), and just be consistent with the age of the universe in the case of extended sources at the highest redshifts. Since there is the possibility that the older populations of synchrotron electrons are polluted by reaccelerated ones or mixed with younger populations in backflow regions, synchrotron ages indicate lower limits. Adopting $100 \mathrm{Myr}$ as fiducial upper limit for radio galaxy lifetimes, Parma et al. (1999) arrive at a typical head advance speed of $v_{\text {head }}=(0.5-5.0) \mathrm{kpc} / \mathrm{Myr}$ for the low power sources. It is not unreasonable that the same parameters apply for high power ones. For those, Scheuer (1995) gives an upper limit of $v_{\text {head }}=30 \mathrm{kpc} / \mathrm{Myr}$, from jet-to-counterjet length asymmetry measurements.

The advance speed of the bow shock in jet direction can be computed from the one-dimensional momentum balance:

$\frac{v_{\mathrm{j}}}{v_{\mathrm{b}}}=\frac{1}{\sqrt{\eta \epsilon}} \sqrt{\frac{1+1 / \gamma_{\mathrm{m}} M_{\mathrm{b}}}{1+1 / \gamma_{\mathrm{j}} M_{\mathrm{j}}}}+1$.

Here, $\epsilon$ is the ratio of beam to head cross section (simulations determine this parameter to $\epsilon \in[0.1 ; 1]$ for $\eta>0.01$ (e.g. Norman et al. 1983), $v_{\mathrm{b}}$ and $M_{\mathrm{b}}$ are velocity and Mach number (with respect to external medium) of the bow shock. $\epsilon$ is a measure for the propagation efficiency. $\gamma_{\mathrm{m}}$ and $\gamma_{\mathrm{j}}$ are the adiabatic indices of external medium and jet beam, respectively. Equation (1) can be approximated for high Mach numbers and low jet density by

$v_{\text {head }}=\sqrt{\eta \epsilon} v_{\mathrm{j}}$

$\eta$ is constrained by the fact that radio lobes develop and the above considerations (Eq. (2)): $3 \times 10^{-6}<\eta<1$. However, it will be shown below that Eq. (2) is no longer valid at these low jet densities. A lower limit of $10^{-7}$ for the density contrast is derived in Sect. 6. Unfortunately, the temperature in the jet beams is not known. Therefore, Mach numbers are unconstrained, observationally.

Jet radii $\left(r_{\mathrm{j}}\right)$ are of the order of kpc (e.g. Carilli \& Barthel 1996; Scheck et al. 2002). From the total radio luminosities, one can estimate the total kinetic jet luminosity. Since the source needs at least the energy to inflate the radio lobes, the total power has to be at least about twice the radio power (for Cygnus A, Carilli \& Barthel 1996), probably more. The most powerful sources at high redshift should therefore have $L_{\text {kin }}>10^{46} \mathrm{erg} / \mathrm{s}$.

\section{Simulation setup}

For an investigation of the parameter space of very light jets, axisymmetric hydrodynamic simulations were performed. The Newtonian 3D MHD code NIRVANA (Ziegler \& Yorke 1997) was used for the computations. NIRVANA is second order accurate and employs a monotonic upwind differencing scheme. It treats the following standard set of hydrodynamic equations:

$$
\begin{aligned}
& \frac{\partial \rho}{\partial t}+\nabla \cdot(\rho \boldsymbol{v})=0 \\
& \frac{\partial \rho \boldsymbol{v}}{\partial t}+\nabla \cdot(\rho \boldsymbol{v} \boldsymbol{v})=-\nabla p
\end{aligned}
$$


$\frac{\partial e}{\partial t}+\nabla \cdot(e \boldsymbol{v})=-p \nabla \cdot \boldsymbol{v}$,

where $\rho$ denotes the density, $e$ internal energy density, $\boldsymbol{v}$ velocity, and $p=(\gamma-1) e$ the pressure. Here $\gamma=5 / 3$ for a nonrelativistic gas is assumed.

For a first scan of the parameter space 12 axisymmetric simulations were performed. The jets were injected in pressure equilibrium into a homogeneous external medium.

Table 1. Simulation parameters.

\begin{tabular}{cccc}
\hline \hline$\eta$ & $v_{\mathrm{j}}[\mathrm{kpc} / \mathrm{Myr}]$ & $M_{\mathrm{int}}$ & $M_{\text {ext }}$ \\
\hline $10^{-2}$ & 95 & 25.56 & 255.6 \\
$10^{-2}$ & 31 & 8.083 & 80.83 \\
$10^{-2}$ & 9.5 & 2.556 & 25.56 \\
\hline $10^{-3}$ & 95 & 25,56 & 808.3 \\
$10^{-3}$ & 31 & 8.083 & 255.6 \\
$10^{-3}$ & 9.5 & 2.556 & 80.83 \\
\hline $10^{-4}$ & 95 & 25.56 & 2556 \\
$10^{-4}$ & 31 & 8.083 & 808.3 \\
$10^{-4}$ & 9.5 & 2.556 & 255.6 \\
\hline $10^{-5}$ & 95 & 25.56 & 8083 \\
$10^{-5}$ & 31 & 8.083 & 2556 \\
$10^{-5}$ & 9.5 & 2.556 & 808.3 \\
\hline
\end{tabular}

The hydrodynamic simulations are fully determined by the internal Mach number $M_{\text {int }}$, the density ratio $\eta$, and the pressure ratio, which is unity. Hence, they are scalable to the parameter range needed in a specific source. Table 1 gives Mach number $(M)$, density contrast $(\eta)$, and the applied velocity. The density in the external medium was set to $2 \mathrm{~cm}^{-3}$, and the jet radius to $r_{\mathrm{j}}=1 \mathrm{kpc}^{1}$. The grid of [600 $\left.\times 600\right]$ points covered an area of $Z \times R=30 \times 30 \mathrm{kpc}^{2}$, so the jet radius corresponds to 20 grid points. Boundary conditions were set to axial symmetry on the axis, reflecting on the left-hand side, and open on the other sides. The reflecting boundary on the left-hand side is essential. If there was an open boundary, a substantial amount of energy would leave the grid on that side. The simulation was stopped when one of the following events happened:

1. The jet propagated to the right-hand side of the grid.

2. The computation time exceeded a reasonable amount without promising new behaviour in the near future. Typically, this time was several weeks.

3. The jet stopped at the nozzle, producing a shock at the inlet which ignored the shock jump conditions. This was caused by entrained material from the shocked external medium approaching the nozzle.

\section{Results}

\subsection{Cocoons}

Contour plots of the final timestep for each simulation are shown in Fig. 1. As expected from higher $\eta$ simulations, the

\footnotetext{
1 The scaling was chosen in order to be easily comparable to previous work, which was adapted to parameters assumed to be present at high redshift (Krause 2002).
}

cocoons broaden with lower $\eta$. Begelman \& Cioffi (1989) derive an analytic formula for the cocoon width $r_{\text {coc }}$ :

$r_{\mathrm{coc}} / r_{\mathrm{j}} \approx 0.7 M_{\mathrm{j}} \eta^{-1 / 4}$.

Figure 1 shows that only the $(M, \eta)=\left(2.6,10^{-2}\right)$ cocoon can be described properly by the above formula. For the other jets, the bow shocks are still too small to contain cocoons of the predicted size. Also, the geometry of the cocoon is quite different from the cylindrical one known from higher $\eta$ jets. The density distributions show more or less spherical cocoons. Between $\eta=10^{-2}$ and $\eta=10^{-3}$, the cocoon undergoes a transition. Sometimes, the vortices it dissolves in are stringed in a line around the beam, but sometimes they join together forming a big vortex which extends approximately over the same size as the jet. Figure $1(M, \eta)=\left(2.6,10^{-3}\right)$ shows a new vortex just before being swallowed by the big one. A vortex with substructure is present in $(M, \eta)=\left(8,10^{-3}\right)$. The interface between cocoon and shocked external medium suffers from Kelvin-Helmholtz $(\mathrm{KH})$ instabilities. They start at small size in the vicinity of the jet head. As they grow, extending their fingers into the cocoon, the backflow accelerates them towards the center. This behaviour is known from higher $\eta$ simulations (Krause \& Camenzind 2001) at high resolution. On the lefthand boundary, the fingers can get long enough to interact with the beam directly. In some cases, this caused a strong shock at the jet inflow, efficiently pushing the jet out of the computational domain (one of the reasons, why the simulation had to be stopped, see above). Quite often, this gas is drawn towards the jet head in between cocoon and jet beam, thus separating the cocoon from the beam. In the $\eta=10^{-5}$ simulations, the initial conditions are not yet relaxed enough to determine the cocoon morphology reliably.

\subsection{Bow shocks}

The bow shocks in the presented simulations are quite different from one another and from simulations with higher $\eta$. Morphologically, they can be classified in three groups. The extremes are located in the upper corners and the bottom row of Fig. 1. The jet in the upper right corner $(M, \eta)=\left(26,10^{-2}\right)$ shows an elongated bow shock, comparable to the simulation of Loken et al. (1992). Due to the high Mach number, the compression ratio is four over the whole surface of the bow shock. Reducing the Mach number to $(M, \eta)=\left(2.6,10^{-2}\right)$, the bow shock gets very regular (upper left corner). Evidently, this is caused by the lag of the contact discontinuity behind the bow shock. Even though there are some disturbances in the shocked external medium, they are not fast enough to catch the leading edge. The bow shock is still stronger in the direction of the jet propagation which produces a higher compression ratio near the head.

If one would reduce the Mach number below one, no jet would form at all, and a spherical sound wave would propagate outwards. In the bottom row $\left(\eta=10^{-5}\right)$, the bow shock is spherical, reminding one of a spherical wave. Nethertheless, one can clearly tell from the density jumps that it is truly a bow shock. So, why is it spherical? A hint comes from the pressure versus number density (pn) histogram (Fig. 5). From their start 

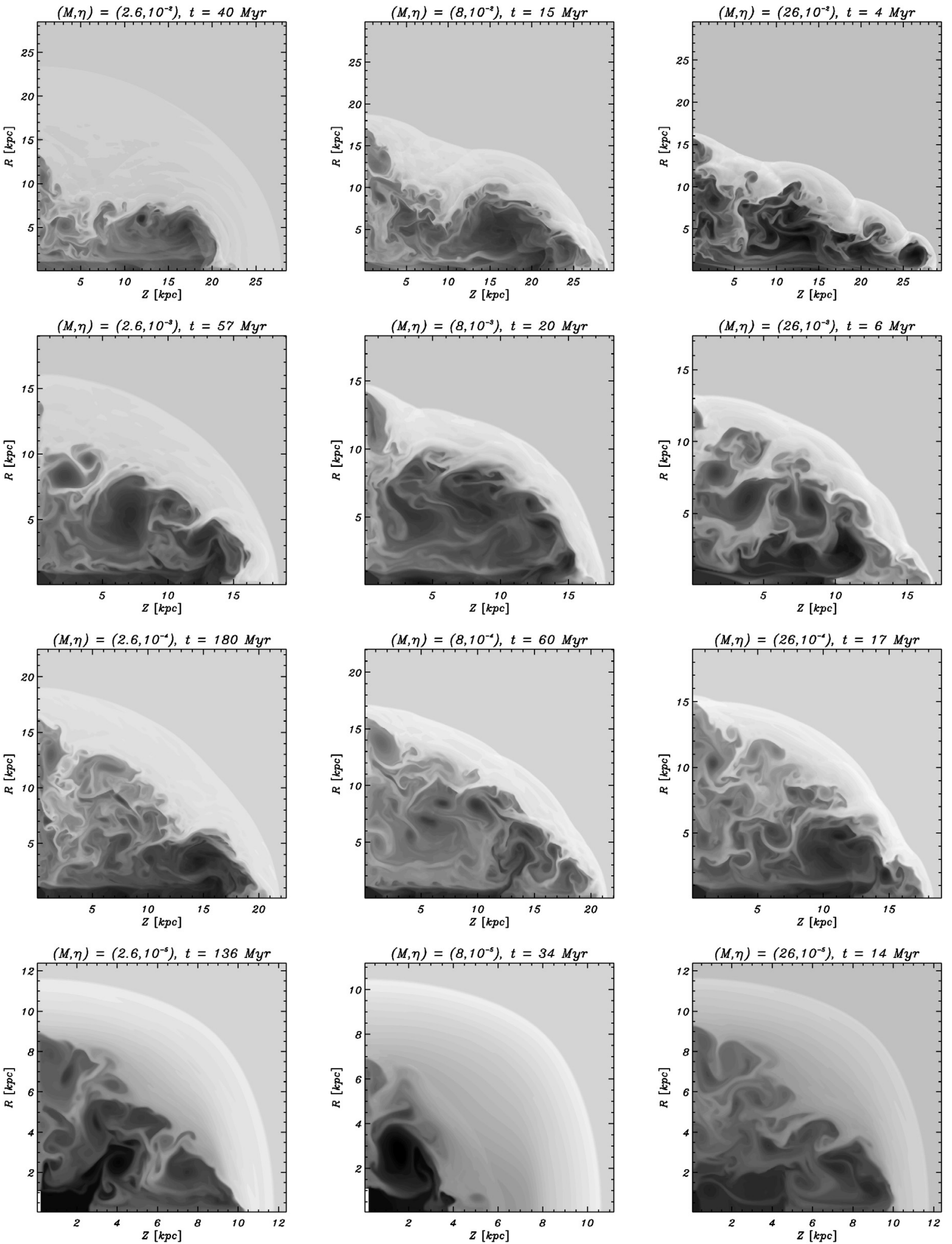

Fig. 1. Overview over the 12 simulations performed in order to scan the parameter space. The Mach number $(M)$ varies from 2.6 to 26 from left to right. Density contrast ranges from $10^{-2}$ (top) to $10^{-5}$ (bottom). The logarithmic density contours vary from dark (low) to white (high). Several jet inlets are artificially white because of a problem in the plotting routine. In fact, they have the lowest values. The simulation time is indicated on top of the individual plots.

position at the external pressure and the density of the jet and external medium ( $10^{5}$ times jet density), respectively the fluid elements move upwards to a thin line of high constant pres- sure. (For the $(M, \eta)=\left(2.6,10^{-5}\right)$ and $(M, \eta)=\left(8,10^{-5}\right)$ plots, the pressure increases so rapidly in the jet that the counts are too few to show up in the plots.) The pressure equilibrium 

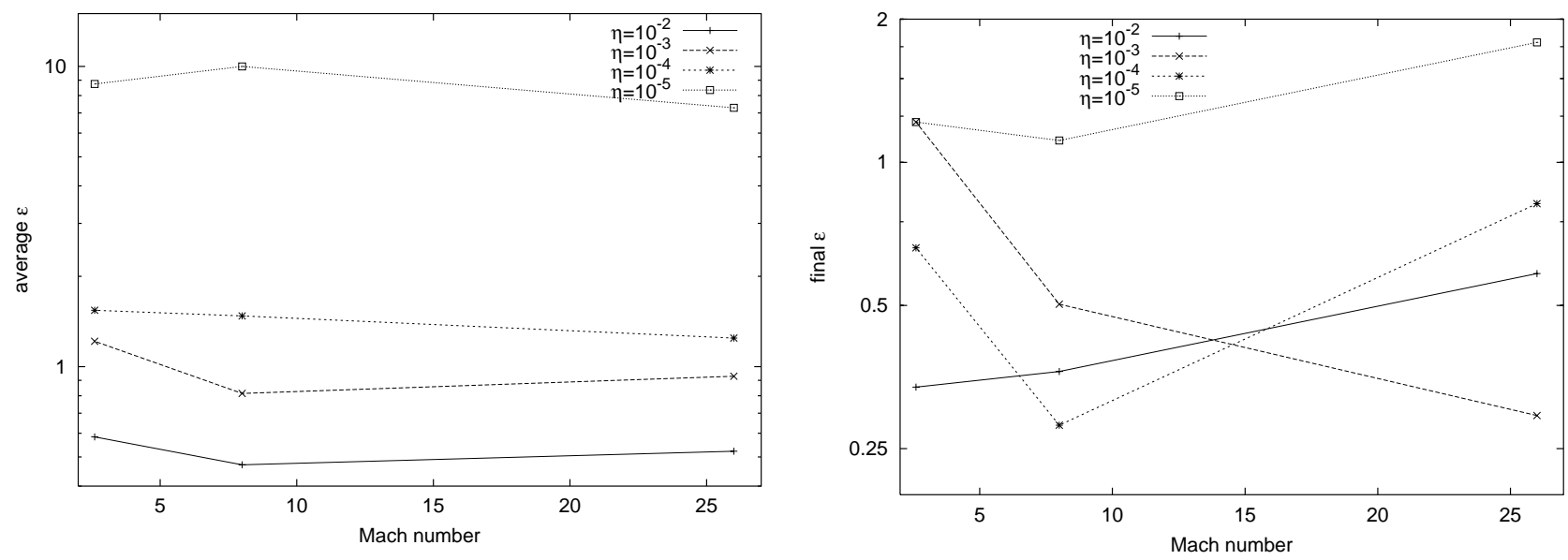

Fig. 2. The propagation efficiency measure $\epsilon$ on average (left panel) and for the last $5 \%$ of the simulation time (right panel).

of all the fluid parts affected by the jet can be understood, if one considers that the sound speed in the jet (roughly $v_{\text {jet }} / 2$ ) is much higher than the bow shock propagation speed. Therefore, sound waves communicate and equalise pressure differences efficiently.

The internal energy within the bubble of the bow shock is sufficient to drive a blastwave. The solution for the blastwave is derived in Sect. 5. In this limit the jet propagates slowly, and mainly transforms its constantly provided kinetic energy into heat. The resulting pressure drives the spherical blastwave. The velocity of the blastwave is proportional to $t^{-2 / 5}$. Hence, the blastwave is faster than the jet bow shock at early times, and slower later on. Equation (12) determines the minimum bow shock radius at which deviation from the spherical blastwave can be expected. For $\eta=10^{-5}$ it turns out to be $9.25 r_{\mathrm{j}}$. The $\eta=10^{-5}$ jets are already slightly above that limit. But the jets are in a quite complicated phase at the time shown in Fig. 1. For example in the case of $(M, \eta)=\left(26,10^{-5}\right)$, one shock in the middle of the jet just became so strong that it developed a backflow there. The old jet head at $Z \approx 9 \mathrm{kpc}$ is dissolving. This is a short phase, and because the blastwave swept much of the matter away, the new jet will soon reach the position of the old one. Then, it will presumably influence the bow shock. That this happens soon after the minimum radius of influence $\left(r_{1}\right)$ is reached, is evident from the $\eta=10^{-4}$ density plots, where $r_{1}=5 r_{\mathrm{j}}$, and from the aspect ratios (see below). One can understand the bow shock shapes of the other simulations as combinations of these three types.

The propagation efficiency measure $\epsilon$ was determined from the simulations on average and for the final $5 \%$ of simulation time. The result is shown in Fig. 2. The highest values of $\epsilon$ are reached for $\eta=10^{-5}$. In that case the bow shock is on average three times faster than the maximum expected from the onedimensional estimate. Again, this shows the high velocity of the blastwave. Also for the $\eta=10^{-4}$ and the $\eta=10^{-3}$ simulations the average efficiency is still high, for the same reason. The $\eta=10^{-2}$ simulations have already reached an extension of roughly 15 times their minimum interaction distance. Therefore $\epsilon$ has fallen considerably below one. Also, it does not change significantly for the final $5 \%$ of the simulation time. Slight variations in the propagation speed are typical for such jets. Extrapolating from higher $\eta$, the accuracy of $\epsilon$ is a few percent for the given resolution (Krause \& Camenzind 2001). Summarising, very light jets blow up a big bubble, rapidly. After reaching $r_{1}$, they stroll along with nonspectacular $\epsilon$.

The aspect ratio of the bow shock was measured in regular intervals, and is shown in Fig. 4 as a function of the position of the bow shock on the $Z$-axis. A general trend is evident for all simulations: up to a propagation distance of two to three jet radii above $r_{1}$ the aspect is approximately one. This is also consistent with the $\eta=10^{-5}$ simulations. Then the aspect starts to rise. This can be explained by considering the bow shock expansion in the axial and radial directions. Up to $r_{1}$, the bow shock has to expand according to the blastwave law, in all directions. After that phase, it changes to a typical jet propagation law, for the axial direction (compare Fig. 3). The jet head sometimes accelerates temporarily, which is well-known in jet physics (beam pumping, compare e.g. Kössl \& Müller 1988), but has on average a constant velocity, given by Eq. (2).

Concerning the radial direction, the bow shock is still pressure driven. It will be shown in Sect. 5 (Eq. (13)) that the Mach number of the bow shock at $r_{1}$ with respect to the external

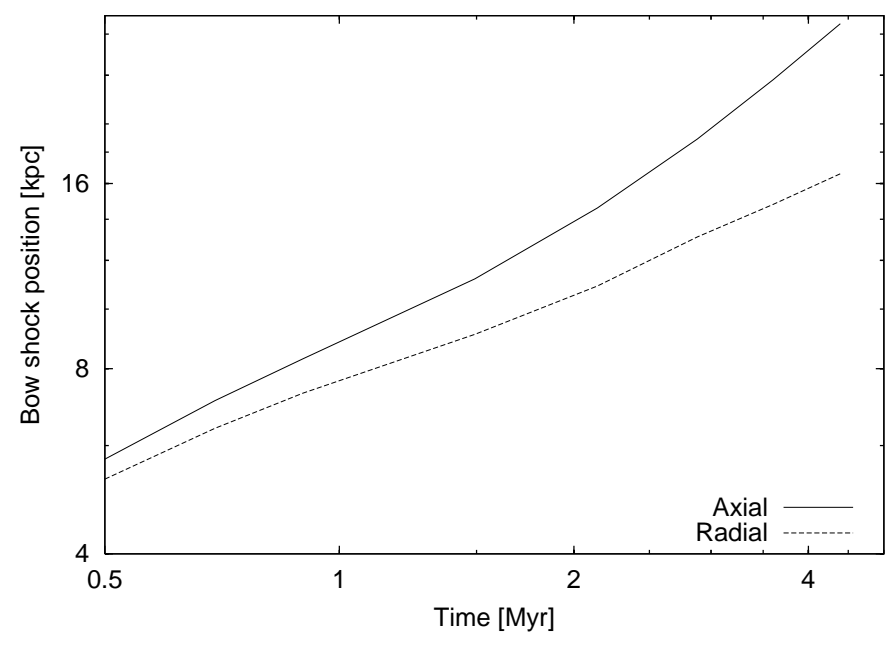

Fig. 3. Bow shock propagation over time for the $(M, \eta)=\left(26,10^{-2}\right)$ simulation. Shown is the position reached on the $R$ and $Z$-axis, respectively. Logarithmic units were used for both axes. 
medium is given by the jet's Mach number $M_{\text {int }}$. Hence, the bow shock is still strong at $r=r_{1}$, at least for the $M>3$ simulations. The deceleration is proportional to the aspect ratio via the pressure (assuming elliptical deformation), and should therefore be higher. On the other hand, secondary shocks in the shocked ambient gas can now reach the surface of the bow shock, accelerating it in limited regions. As an example, for the $(M, \eta)=\left(8,10^{-4}\right)$ simulation, the relative decrease of the radial bow shock velocity $\left(1-v_{\mathrm{b}} / v_{\mathrm{a}}\right)$ between radial bow shock positions $r_{\mathrm{a}}=6.65 \mathrm{kpc}$ and $r_{\mathrm{b}}=17.0 \mathrm{kpc}$ amounts to $47.8 \%$. One can easily derive from Eq. (11) that the blastwave law predicts a decrease of only $1-\left(r_{\mathrm{a}} / r_{\mathrm{b}}\right)^{2 / 3}=46.5 \%$. The agreement between the numbers is quite good in this case. In the other simulations there is more accumulation of jet material at the left boundary (compare Fig. 1), which sometimes strongly disturbs the bow shock near the left boundary. The difference between the measured and predicted velocity decrease can reach a factor of two there. However, the boundary condition at the left side complicates the interpretation. A preliminary conclusion is therefore that the radial bow shock expansion keeps following the blastwave law for the simulated jets with $M>3$ and $\eta<10^{-2}$ for the simulated times, if the disturbance by the material accumulated at the left boundary is not too strong. In a follow-up paper, where a 3D simulation with bipolar jets will be presented, this issue will be addressed in more detail.

Consequently, the aspects increase with time. In Fig. 1, the $\eta=10^{-3}$ and $\eta=10^{-4}$ simulations have left the blastwave phase not long ago, whereas the $\eta=10^{-2}$ jets are already more than ten times bigger than $r_{1}$. The former jets are observed to push comparatively gently, basically elongating the bubble. The $\eta=10^{-2}$ jets show an interesting dependence on the Mach number: only the low Mach number jet keeps the elongated spherical shape.

The cocoons of the high Mach number jets are underexpanded according to Eq. (6), and act violently on the bow shocks. In the phase where the bow shock is only slightly elongated, the bow shock velocity in axial direction has not yet reached the constant velocity. This can be seen from Fig. 3: the gradual change between the two propagation laws is finished at $t \approx 2 \mathrm{Myr}$, for the $(M, \eta)=\left(26,10^{-2}\right)$ jet. At that time the bow shock has reached approximately $10 r_{1}$. The limiting aspect ratio $(a)$ can then easily be computed from Eq. (2):

$a=\frac{v_{\mathrm{j}} \sqrt{\epsilon \eta}}{c_{\mathrm{s}, \mathrm{ext}}}=M_{\mathrm{ext}} \sqrt{\epsilon \eta}$,

where the index ext denotes quantities in the external medium. With the values from Table 1 the limiting aspect ratios turn out to be between $1.6(M=2.6)$ and $16(M=26)$. None of the simulations has reached this limit yet.

\subsection{Beams}

A prominent feature of the jet beams is the strong shock roughly one jet radius behind the jet inlet (compare Fig. 6). This indicates the first reflection point of the oblique shocks in the jet. The shock is caused by the high pressure and velocities of the backflow, which act on the beam. The position of the first shock reflection point varies with time. This is consistent
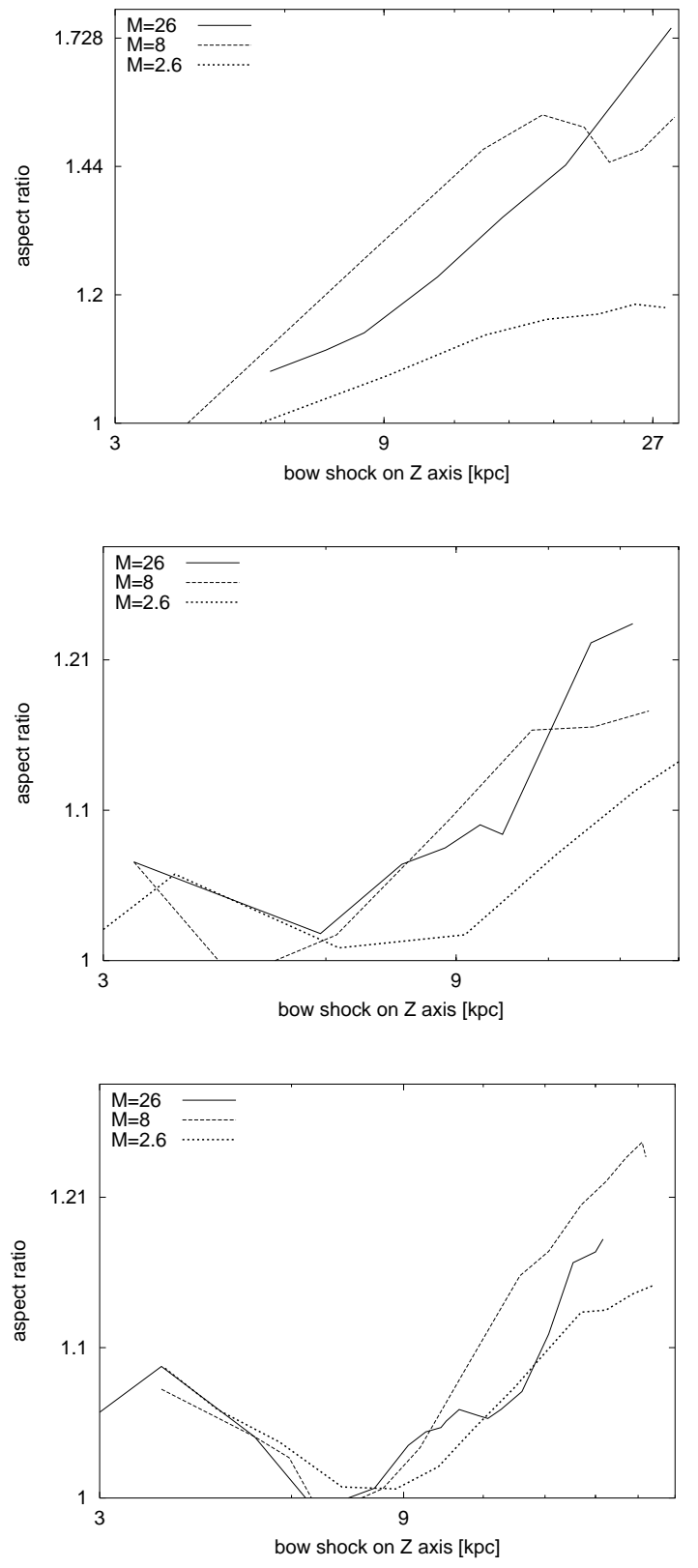

Fig. 4. Aspect ratio of the bow shock over propagation distance. The upper panel shows the $\eta=10^{-2}$ simulations, the middle one the $\eta=10^{-3}$ simulations, and the $\eta=10^{-4}$ simulations are shown in the bottom panel. Logarithmic units were used for both axes.

with simulation results at higher density contrast with closed left boundary conditions (Kössl \& Müller 1988).

High Mach numbers $(M>3)$ can be sustained for the $\eta=10^{-2}$ jets (compare Fig. 6). With the exception of the $(M, \eta)=\left(26,10^{-3}\right)$ jet, all the other beams struggle around $M=1-2$. Quite often they become subsonic, even if they start with high Mach numbers. Strong shocks decelerate those jets. Since also shocked ambient gas reaches the beam quite often, nothing prevents the beams from being disrupted by the $\mathrm{KH}$ instability. A fine example is the $(M, \eta)=\left(26,10^{-3}\right)$ simulation (Fig. 1). The shocks in the beams sometimes get strong enough to shed vortices and produce backflows, thereby establishing a 
new jet head. This behaviour is similar to axisymmetric simulations of higher $\eta$ jets (e.g. Kössl \& Müller 1988; Jones et al. 1999), but seems to be more violent at lower density contrast.

The $\eta=10^{-5}$ jets suffer from the short evolution time. At the time shown, the jets still have the prominent shock at the jet inlet, which was used as initial condition. Since those are the computationally most expensive simulations, the problem can only be solved with a faster computer, evolving the jets longer.

\subsection{Pressure versus number density histograms}

From Fig. 5, it is evident that very light jets provide their own pressure everywhere. External medium as well as jet plasma get strongly shocked, and the high sound speed equalises the pressure within most of the jet bubble, especially in the higher Mach number cases. The external pressure is therefore unimportant, and the pressure matching, used as initial condition, can be dropped in future simulations, without much difference. The pn-histograms show many counts at intermediate densities. They are caused by fluid elements resulting from mixing of jet plasma with shocked external medium. This is present in every simulation, although there are typically less counts than in the region of shocked external medium densities (right) or jet densities (left). Especially prominent are several straight lines in the pn-histograms. They extend to very low densities, present in the center of vortices. The following example examines the $(M, \eta)=\left(2.6,10^{-2}\right)$ jet. The density contours show two prominent vortices (dark) in the cocoon. Correspondingly, the pn-histogram shows two spikes extending down to a tenth of the jet density. Now, two areas, each centered on one vortex were cut out and examined separately. The individual pnhistograms are shown in Fig. 7. Clearly, in the right figure, the upper spike has disappeared. Nevertheless, it is prominent in the left figure. Hence, it is evident that vortices show up in the pn-histograms as straight lines. Therefore they follow the relation $p \propto n^{\Gamma}$. The left and right vortices of Fig. 7 have $\Gamma=0.35$ and $\Gamma=1.26$, respectively. The spikes in the other histograms have a maximum $\Gamma$ of $1.5-1.7$. If a vortex would originate from jet material with uniform density and would be compressed in a shock with uniform compression ratio $\kappa$, it would have a uniform entropy index $s \equiv p / n^{\gamma}$. The pressure differences are caused by adiabatic changes due to the centrifugal force. The consequence is a $p=s n^{\gamma}$ subsystem, which is often observed in the pn histograms. The low $\Gamma$ of the two vortices in the $(M, \eta)=\left(2.6,10^{-2}\right)$ is exceptional. Systems of that kind are occasionally created in a strong shock in the vicinity of the jet axis connected with a temporal on-axis backflow. Such shocks are usually weaker in a 3D simulation. The life time of these systems is short. They mix rapidly with other material due to the limited grid resolution.

The bow shock obeys a curved line in the pn-histogram. This is especially evident from the $(M, \eta)=\left(8,10^{-5}\right)$ simulation. The density contours show most of the space occupied by shocked external medium. In the pn-histogram, these fluid elements, starting at $(\log (n), \log (p)) \approx(5,0)$, first move upwards, bending to the right. The maximum pressure and density in the shock is reached in a rightmost pinnacle $((\log (n), \log (p)) \approx(5.6,2.2))$. Then pressure and density decline fast to the isobaric regime. Here the density declines further. Even in this case, with the smallest cocoon, $87 \%$ of the mass is concentrated in the region $8.5 \mathrm{kpc}<r<10.5 \mathrm{kpc}$, forming a thin shell.

\section{Analytic approximation for the blastwave phase}

In the simulations of the last section the bow shock was found to resemble a spherical blastwave up to a certain time. The simulations show that the cocoons displace most of the external medium into a shell. Assuming a constant velocity $v$ for a shell of mass $\mathcal{M}$, one can find an analytic approximation for the expansion of this shell by postulating force balance at the bow shock surface:

$\frac{\partial}{\partial t}(\mathcal{M} v)=S\left(p_{\text {int }}-p_{\text {ext }}\right)$.

Here, $S=4 \pi r^{2}$, and $p_{\text {int }}$ and $p_{\text {ext }}$ denote internal and external pressure, respectively. The internal pressure is given by $p_{\text {int }}=$ $\frac{2}{3}\left(E(t)-\mathcal{M} v^{2} / 2\right) /\left(4 \pi r^{3} / 3\right)$, where $E(t)$ is the energy injected into the cocoon by the jet.

Usually one considers self-similar solutions of the spherical force balance (e.g. Heinz et al. 1998; Soker et al. 2002; Krause 2002). Here, an alternative approach is presented, computing the global integral of the equation.

\subsection{General solution}

In the case of negligible external pressure, which is the case for strong bow shocks, Eq. (8) can be integrated:

$\int_{0}^{r} \mathcal{M}(r) r \mathrm{~d} r=2 \int_{0}^{t} \mathrm{~d} t_{1} \int_{0}^{t_{1}} E\left(t_{2}\right) \mathrm{d} t_{2}$.

Equation (9) is the general solution for any sort of energy input driving a blastwave into a medium with arbitrary density distribution, as long as both are integrable functions.

\subsection{Power law solutions}

A very useful type of solutions is found, if one assumes energy and density to be given by power laws:

$E=\mathcal{L} t^{d}$

$\rho=\rho_{0}\left(r / r_{0}\right)^{\kappa}$.

The solution for the shell radius is in that case:

$r=\left(\frac{(\kappa+3)(\kappa+5)}{(d+1)(d+2)} \frac{r_{0}^{\kappa} \mathcal{L}}{2 \pi \rho_{0}}\right)^{\frac{1}{\kappa+5}} t^{\frac{d+2}{\kappa+5}}$.

For example, setting total energy and background density to a constant $(\kappa=d=0)$ gives the well-known solution for expanding supernova bubbles (Sedov 1959). Jets can be assumed to deliver a constant amount of energy to their cocoons. Therefore $d=1$ is appropriate. With that choice, the functional dependence of the self-similar expansion law from Heinz et al. (1998) is recovered. However, for density exponents between -2 and 0 , the constant of proportionality is by a factor of 4 

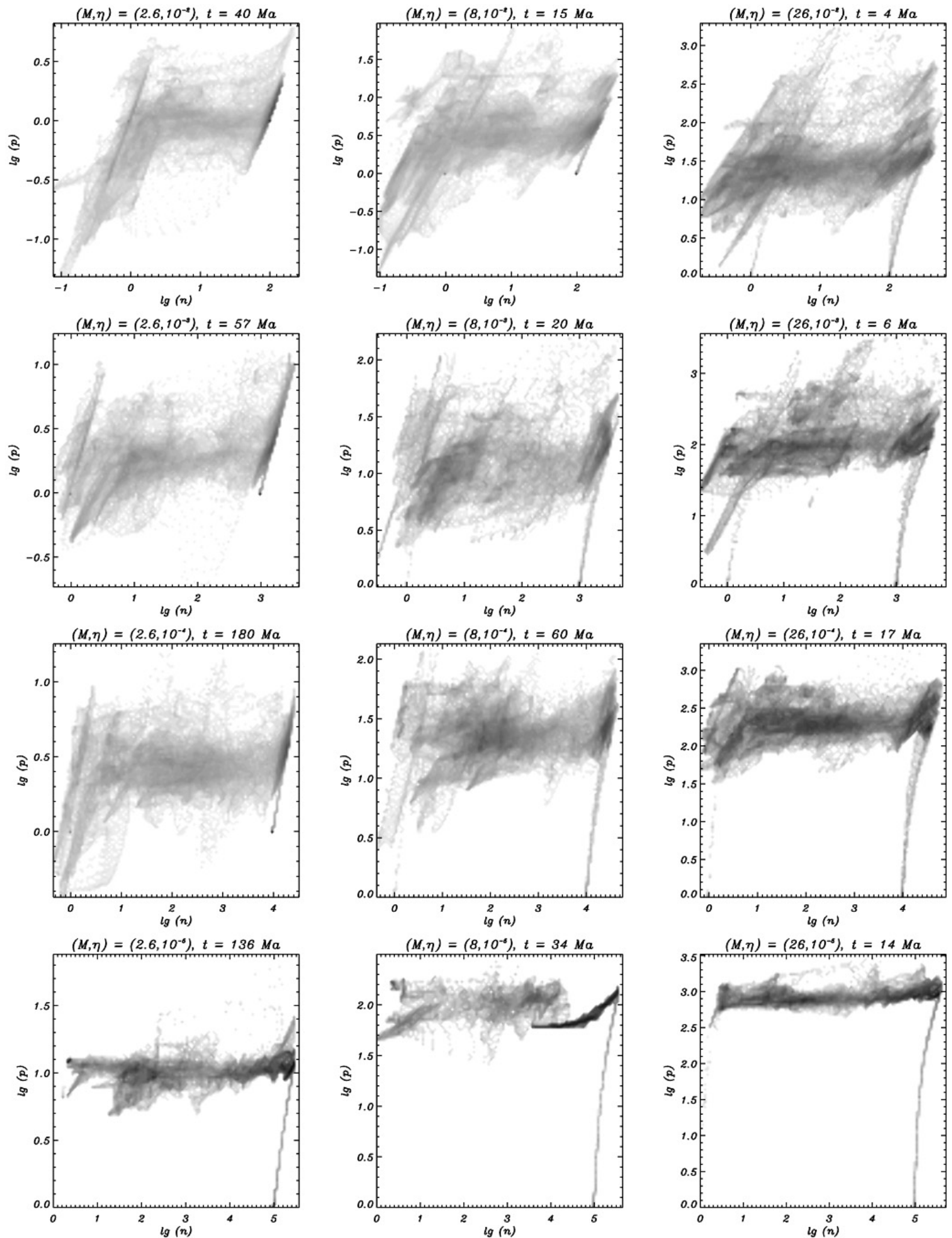

Fig. 5. Pressure over number density (pn) histograms. 100 bins were used on each axis. Pressure, number density, and counts are given in logarithmic units. Darker regions have more counts. Pressure and density have been normalised to the initial jet values.

to 1 lower in the present solution. This reflects the fact that it is, from the mathematical point of view, a global solution. Therefore, for density exponents lower than -3 the bubble has to fight against an infinite amount of mass at $r=0$. Equation (9) consequently states that the blastwave will not expand at all. This is of course not found in a self-similar solution, since such a solution is always assumed to be far from the boundaries. A relativistic gas in the cocoon, as taken into account by 

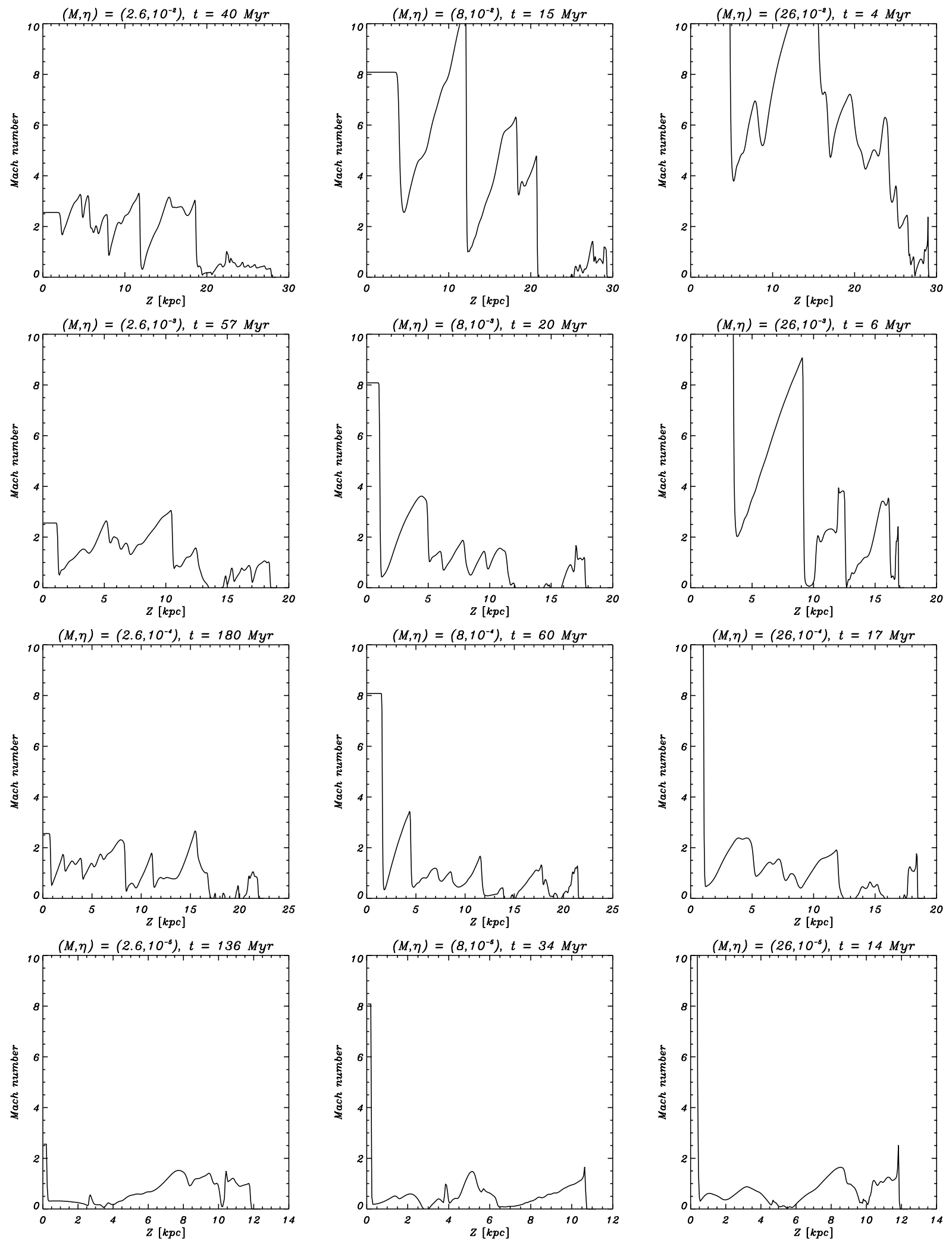

Fig. 6. On-axis Mach number.

Heinz et al. (1998), increases the constant of proportionality by roughly $10 \%$. Because the blastwave is decelerating for $\kappa>-2$, whereas the bow shock from a jet should propagate at approximately constant speed (compare Eq. (2)), the bow shock is first spherical, and gets shaped by the jet only if the jet thrust can push it faster. The expansion velocity of the blastwave $v$ from a non-relativistic twin jet is given by:

$$
\left(\frac{v}{v_{\mathrm{j}}}\right)^{3}=\frac{9}{4} \frac{\kappa+3}{(\kappa+5)^{2}} \eta_{0}\left(\frac{r}{r_{0}}\right)^{-\kappa}\left(\frac{r}{r_{j}}\right)^{-2},
$$



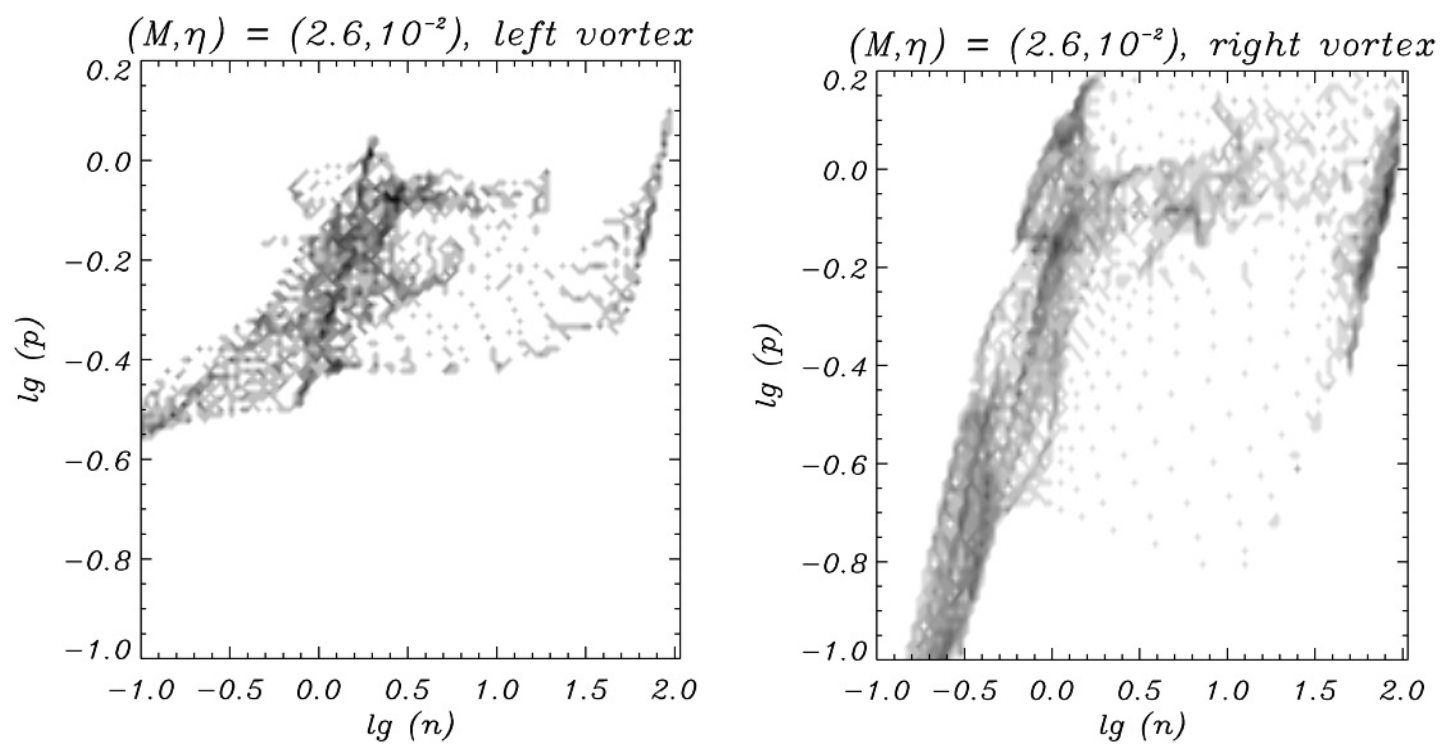

Fig. 7. $p n$-histograms for regions extracted around the two vortices of the $(M, \eta)=\left(2.6,10^{-2}\right)$ jet. The left figure corresponds to the left vortex.

where $\eta_{0}=\rho_{\mathrm{j}} / \rho_{0}$. Combining this with Eq. (2), and choosing $r_{0}=r_{\mathrm{j}}$, results in:

$\frac{r_{1}}{r_{\mathrm{j}}}=\left(\frac{16}{81} \frac{(\kappa+5)^{4}}{(\kappa+3)^{2}} \eta_{0} \epsilon^{3}\right)^{\frac{1}{\kappa-4}} \approx 0.5\left(\eta_{0} \epsilon^{3}\right)^{\frac{1}{\kappa-4}}$

$r_{1}$ denotes the radius at which the jet first affects the bow shock. The latter approximation is valid for $0>\kappa>-2.9$ with an accuracy of $25 \%$. The coefficient goes to zero for $\kappa$ towards -3 . The minimum $r_{1}$ is reached for $\epsilon=1$. It may be surprising at the first glance that the jet head can even more easily reach accelerating bubbles. This is due to the fact that the jet head accelerates always faster than the blastwave, as long as the assumptions leading to Eq. (2) are appropriate. It follows that spherical bow shocks are expected for very light jets, in small systems, and in environments where the density does not decrease faster than with $\kappa \approx-2.9$. The latter restriction does not apply, if the density exponent turns smoothly into -3 , which is demonstrated below.

The assumption of negligible external pressure holds as long as the bow shock is much faster than the sound speed in the external medium, which can also be shown explicitely from the equations in this section. The bow shock has a Mach number $M_{\mathrm{B}}$ with respect to the external medium at a radius $r$, in the isothermal case given by

$\frac{r}{r_{1}}=\left(\frac{9}{4} \frac{\kappa+3}{(\kappa+5)^{2}} \eta_{0}^{-2 / \kappa}\right)^{\frac{3 \kappa}{\kappa^{2}-2 \kappa-8}} \epsilon^{\frac{3}{4-\kappa}}\left(\frac{M_{\mathrm{ext}}}{M_{\mathrm{B}}}\right)^{\frac{3}{\kappa+2}}$.

For the conditions of the simulations presented here $(\kappa=0)$, Eq. (13) states that the Mach number of the bow shock exceeds the jet Mach number when the bow shock reaches $r_{1}$. If $\kappa<0$, the bow shock Mach number at $r=r_{1}$ is even higher for $\eta_{0}<10^{-2}$. It can reach typically several times the jet Mach number. In an isobaric atmosphere, the relation becomes:

$$
\frac{r}{r_{1}}=\left(\delta \epsilon\left(\frac{M_{\mathrm{int}}}{M_{\mathrm{B}}}\right)^{2}\right)^{\frac{3}{4-\kappa}}
$$

where $\delta$ is the overpressure ratio of the jet. Again, the bow shock is supersonic at $r_{1}$ for supersonic jets, unless they are heavily underpressured.

\subsection{King type solutions}

Extragalactic jets often propagate into environments where the density cannot be approximated by a single power law. A King type density distribution is often used for density distributions around galaxies:

$\rho(r)=\rho_{0}\left(1+\left(\frac{r}{r_{0}}\right)^{2}\right)^{-3 \beta / 2}$.

The integrals in Eq. (9) can be performed analytically for some cases of $\beta$, resulting in:

$t=\sqrt[3]{\frac{12 \pi \rho_{0} r_{0}^{5}}{\mathcal{L}} Y}$,

where $Y$ is given by

$Y=\left\{\begin{array}{l}\frac{1}{8} x\left(A^{3}+A / 2\right)-\frac{1}{4} \operatorname{arcsinh}(x) B \\ \frac{1}{2}\left(\frac{2}{3} x C-\arctan (x) A^{2}\right) \\ \frac{1}{2} \operatorname{arcsinh}(x) C-\frac{3}{4} x A\end{array}\right\}$ for $3 \beta=\left\{\begin{array}{l}1 \\ 2, \\ 3\end{array}\right.$

with

$A=\sqrt{1+x^{2}}$

$B=3 / 4+x^{2}$,

$C=3 / 2+x^{2}$, and

$x=r / r_{0}$.

The solutions from Eq. (17) are plotted in Fig. 8. It is evident that the effect of a King atmosphere, which is a smooth transformation from a constant density profile to a decline with exponent $-3 \beta$, transforms the corresponding power law solutions smoothly into one another. For $\beta=2 / 3$, which corresponds to 


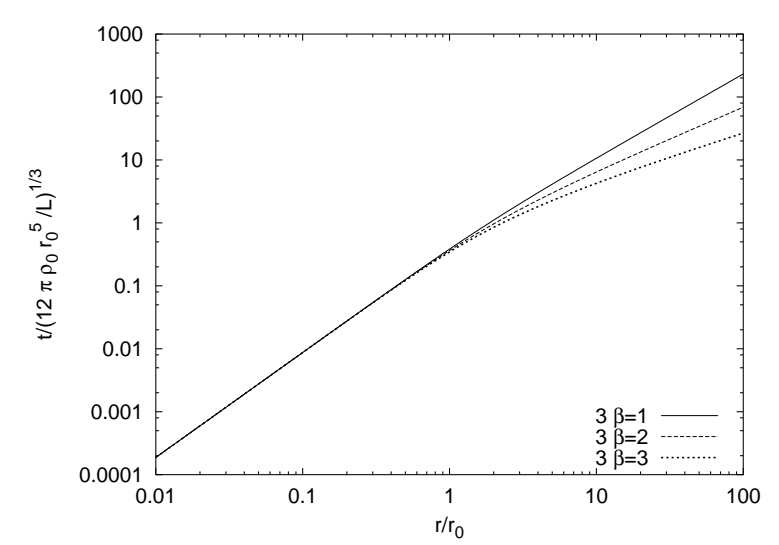

Fig. 8. Propagation of a blastwave powered by a jet into a King type atmosphere with $\beta$ as indicated above.

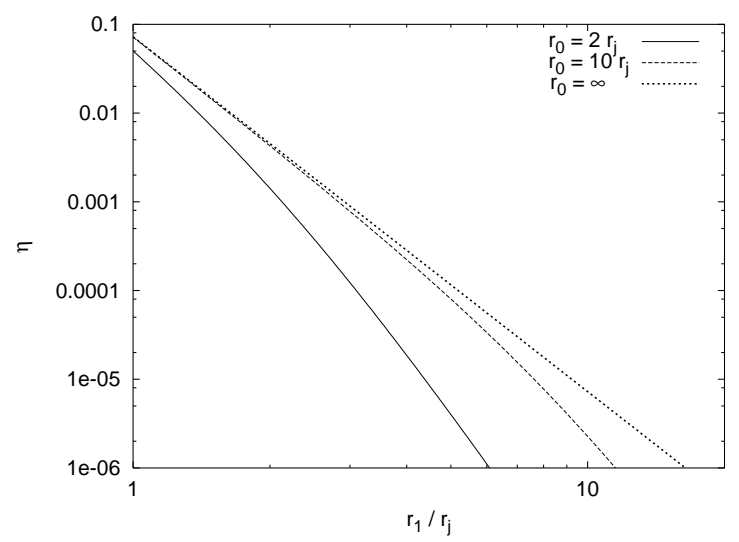

Fig. 9. Minimum radius of jet influence $\left(r_{1} / r_{\mathrm{j}}\right)$ (horizontal axis) as a function of density contrast $\eta=\rho_{\mathrm{j}} / \rho_{0}$ for different core radii $r_{0}$ in a $\beta=1$ King profile. For $r_{0}=\infty$ the density is constant.

a $\kappa=-2$ power law solution for large $x$, the shell reaches constant velocity at infinity. For steeper density declines, the shell accelerates. In that case it is Rayleigh-Taylor unstable, and considerable mixing with the cocoon can be expected, as already pointed out by Heinz et al. (1998). In the same manner as for the power law case, one can compute $r_{1}$ for the King density distributions. For example, in the $\beta=1$ case $r_{1}$ is given by the following formula:

$\eta \epsilon^{3}=\left(\frac{3}{4}\right)^{4}\left(\frac{r_{\mathrm{j}}}{r_{0}}\right)^{4}\left(1+x_{1}^{2}\right)^{-9 / 2} D\left(x_{1}\right)^{-6}$,

where

$x_{1}=r_{1} / r_{0}$,

$D\left(x_{1}\right)=E\left(x_{1}\right)^{-2 / 3} F\left(x_{1}\right)$,

$E\left(x_{1}\right)=\operatorname{arcsinh}\left(x_{1}\right) C\left(x_{1}\right)-\frac{3}{2} x_{1} A\left(x_{1}\right)$, and

$F\left(x_{1}\right)=x_{1} \operatorname{arcsinh}\left(x_{1}\right)-x_{1}^{2} / A\left(x_{1}\right)$.

$r_{1}$ is plotted for three different core radii in Fig. 9. This density profile tends towards $r^{-3}$ for large $r$. As predicted above, $r_{1}$ is finite also in that density regime. For $\eta>10^{-4}$ and $r_{0}>10 r_{\mathrm{j}}$, the result is not much different from the constant density case. But in the more extreme cases $r_{1}$ can be reduced considerably, e.g. for $\eta=10^{-6}, r_{0}=2 r_{\mathrm{j}}, r_{1}$ is reduced from 16 to 6 jet radii.
Concerning the propagation law, the constant density formula approximates the King solution to $10 \%$ up to approximately one core radius for the shallowest density profile and half a core radius for the steepest one (Fig. 8).

Summarising, the constant density formulas can be used to approximate the true solutions if $r_{1}$ is not much bigger than the core radius, if the density contrast is above $10^{-4}$, and the density profile steepens not much more than into a $r^{-2}$ power law.

\section{Discussion}

A very interesting feature of the above results is the blastwave phase. Since the blastwave is faster at early times, it paves the way for the jet, effectively increasing the jet propagation velocity. Later, the jet head is faster than the blastwave equation predicts. Therefore, an upper limit for jet ages is given by Eq. (10). This can be used to establish a lower limit for $\eta$. For very light jets, one can regard $r_{1}$ as a typical extention. The time for a jet to reach the distance $r_{1}$ in a constant density atmosphere is:

$t\left(r_{1}\right)=\frac{1}{2 \sqrt[3]{5}} \eta^{-3 / 4} r_{\mathrm{j}} / v_{\mathrm{j}}$

With the observational constraints cited in Sect. 2, one obtains a new lower limit for the density contrast in extragalactic jets: $\eta>10^{-7}$.

It was shown that the aspect ratio of the bow shock depends on the source size. It is close to unity in the blastwave dominated phase, and starts to increase soon after a critical size $\left(r_{1}\right)$ is reached. This increase will come to an end when the sideways expansion velocity drops to the sound speed. Saxton et al. (2002) have computed similar models with density contrasts down to $\eta=10^{-4}$. The simulation results are generally comparable in the regions where the simulation parameters overlap. The aspect ratio at the end of their $\eta=10^{-4}$ simulation is also close to 1.2. The aspect ratio can be used to constrain density ratios. For example, the aspect ratio of 1.2 for the bow shock in Cygnus A (Smith et al. 2002) together with its extension of roughly $120 r_{\mathrm{j}}$ (Carilli \& Barthel 1996) are clearly inconsistent with $\eta \geq 10^{-2}$. Probably, $\eta$ should be below $10^{-3}$. With cluster temperatures of approximately $10^{8} \mathrm{~K}$ (Smith et al. 2002), resulting in probable external Mach numbers of a few hundred, one derives a limiting aspect ratio of about 2, which the source has not yet reached. However, the jet clearly acts on the bow shock, and therefore one can check Eq. (12), which is fulfilled if $\eta>3 \times 10^{-6}$. Another result was that low cocoon width to bow shock width ratios can only be achieved in low internal Mach number jets. This result is depends crucially on the closed left boundary, which is essential in order to keep the internal energy inside of the computational domain. Other studies have shown that the cocoon can be small even in high Mach number jets for an open boundary on the injection side (Carvalho \& O'Dea 2002a; Saxton et al. 2002). Applying Eq. (6) results in a probable range of $\eta \in\left[10^{-5} ; 10^{-3}\right]$ for $M \in[2 ; 6]$ for the jet in Cygnus A.

With the general solution of the spherical force balance, one can also predict how the results achieved here change in a nonhomogeneous density distribution. It was shown that - for a King profile - the blastwave phase is a little shorter for very 
small core radii. But in most real cases, the blastwave phase will remain up to similar source sizes as in the constant density case. This will be similar in all profiles with a constant density in the central region. Preliminary results from simulations with a King density distribution support this conclusion.

The presented results are subject to two potentially important limitations: The axisymmetry and the left hand boundary. 3D simulations of light jets have shown that big vortices are unlikely to survive (Tregillis et al. 2001). One should therefore expect turbulence on smaller scales in the 3D case. The jet beams should be less disturbed, and might be able to sustain higher Mach numbers. So, they might influence the bow shock earlier than the 2D jets. However, since the 2D jets show this interaction already shortly after reaching the theoretical $r_{1}$, the difference should not be severe in that respect. The left boundary is an obstacle for material that would otherwise leave the grid there, and interact with the counterjet. This should change the appearance of the cocoon in regions around $Z=0$. Both these issues will be addressed in a future paper where a $3 \mathrm{D}$ simulation with bipolar (back-to-back) jets will be presented.

By now, more and more X-ray bubbles are detected around radio sources, mainly in CD galaxies (e.g. Soker et al. 2002, and references therein). Such bubbles can be interpreted as gas contained within the bow shock (or the bow wave, if the shock has relaxed) from the jet. The theory presented in this work should be applicable to some of those sources. The typical bubble size for which one should expect supersonic bow shocks (homogeneous atmosphere) is given by:

$$
r=24\left[\frac{0.1 \mathrm{~cm}^{-3}}{n_{0}} \frac{\mathcal{L}}{10^{46} \mathrm{erg} / \mathrm{s}}\left(\frac{1000 \mathrm{~km} \mathrm{~s}^{-1}}{c_{\mathrm{s}}}\right)^{3}\right]^{1 / 2} \mathrm{kpc},
$$

for typical values of the sound speed $c_{\mathrm{s}}$ and number density $n_{0}$ in the external medium. We should therefore expect no strong bow shocks around jet sources with tens of $\mathrm{kpc}$ or more in diameter (compare e.g. in Hydra A, Nulsen et al. 2002). This conclusion is not changed if one considers elongated bubbles, since the increased volume due to the elongation can only decrease the pressure inside the bubble. Hence, Eq. (20) gives an upper limit. This means that the main energy transfer from radio sources to the gas of a galaxy cluster happens at early times in the jet evolution. The power input into the cluster gas is $30 \%$ of the total jet power for the constant density case. This follows from integration of the energy gain at the bow shock. A strong jet source therefore has delivered

$$
\begin{aligned}
E_{\text {gas }}= & 10^{60} \mathrm{erg}\left(\frac{\mathcal{L}}{10^{46} \mathrm{erg} / \mathrm{s}}\right)^{3 / 2}\left(\frac{v_{\mathrm{j}}}{c / 3}\right)^{-5 / 2} \\
& \times\left(\frac{\eta}{10^{-4}}\right)^{-5 / 4}\left(\frac{n_{0}}{0.1 \mathrm{~cm}^{-3}}\right)^{-1 / 2}
\end{aligned}
$$

to its surroundings when its bow shock reaches $r_{1}$. This is already a considerable fraction of the total energy of the gas in a typical galaxy cluster. Cooling flows can therefore easily be stopped by a powerful jet. One such episode every $1000 \mathrm{Myr}$ could balance the energy loss and keep the cluster temperature in the observed $\mathrm{keV}$ range.

The results suggest that spherical bubbles should be found around most of the extragalactic jet sources at early evolutionary stages. The classes of compact symmetric objects (CSO) and GHz peaked sources (GPS) are associated with such sources. Unfortunately, the bubbles are quite small. In order to resolve a region of a few kpc in diameter with Chandra, the source has to have a redshift considerably below one, where few objects where found (Snellen \& Schilizzi 2002). The hot $\left(10^{9} \mathrm{~K}\right)$ bubble would radiate in the X-rays at about $10^{41} \mathrm{erg} / \mathrm{s}$, hard to discern from a quasar background.

What about the radio morphology? As was shown above, young jets in the bubble phase are often disturbed or disrupted. This is also true for CSO/GPS sources (Saikia et al. 2002). They are e.g. much more asymmetric than large scale jets. An old puzzle with CSO/GPS sources was if they were stuck within their galaxy because of an especially dense interstellar medium (ISM) or because they are young. With the results obtained above, one can exclude the first possibility. Let us consider for example a jet with a power of $10^{47} \mathrm{erg} / \mathrm{s}$ propagating at near light speed through an incredibly dense ISM, $10^{8}$ times denser than the jet. For the first kpc, a typical core radius, it would take the jet head about $30 \mathrm{Myr}$, if propelled by the direct thrust alone. Such a jet was probably in the bubble phase for most, or maybe all, of its lifetime. The upper limit on its age from Eq. (10) turns out to be $0.3 \mathrm{Myr}$, even for an ISM density of $100 \mathrm{~cm}^{-3}$. Therefore, the probability of finding a source at a small angular size is not increased by considering dense environments. Recent age determinations by spectral aging methods and VLBI kinematics of hotspots strongly argue for young source ages of CSOs (Conway 2002).

The KH instability at the contact discontinuity is ubiquitous. This is especially interesting since the same behaviour was found for $\eta=10^{-1}$ at highest resolution (Krause \& Camenzind 2001). The KH instability entrains shocked external medium into the jet cocoon, growing and accelerating it towards the center. This mechanism seems to be a promising candidate for bright X-ray features or the production of emission line regions, where the densities are higher (Bicknell et al. 2000): the gas is accelerated and pulled down inside the cocoon, where it can radiate by recombination or reradiation of quasar light. Given the high energy content of the radio lobes, one could also imagine that they somehow transfer energy to the entrained gas. Since the cocoon plasma is quite exotic, the classical heat conduction formulas are probably not appropriate. Therefore, exact computations are beyond the scope of this work.

Concerning the beams, one can fairly say that those nonrelativistic jets without significant magnetic field cannot travel to large distances without being disrupted. One needs a magnetic field in order to keep some of the cocoon around the beam, and to damp the $\mathrm{KH}$ instabilities in the beam. Alternatively, they would always look very disturbed, morphologically, until the bow shock had swept enough material away, so they could effectively travel into a less dense medium. Since bow shock detections point clearly in the direction of strong density contrast (i.e. $\eta \ll 1$ ), a solution to this problem can be expected from further research.

Acknowledgements. This work was supported by the Deutsche Forschungsgemeinschaft (Sonderforschungsbereich 439). Thanks to 
the advice of the referee, Ian Tregillis, this paper has been greatly improved.

\section{References}

Baade, W., \& Minkowski, R. 1954, ApJ, 119, 206

Begelman, M. C., \& Cioffi, D. F. 1989, ApJ, 345, L21

Bicknell, G. V., Sutherland, R. S., van Breugel, W. J. M., et al. 2000, ApJ, 540, 678

Britzen, S., Witzel, A., Krichbaum, T. P., et al. 2000, A\&A, 360, 65

Carilli, C. L., \& Barthel, P. D. 1996, A\&ARv, 7, 1

Carilli, C. L., Perley, R. A., \& Harris, D. E. 1994, MNRAS, 270, 173

Carvalho, J. C., \& O'Dea, C. P. 2002a, ApJS, 141, 337

Carvalho, J. C., \& O'Dea, C. P. 2002b, ApJS, 141, 371

Cioffi, D. F., \& Blondin, J. M. 1992, ApJ, 392, 458

Clarke, D. A. 1993, in Jets in Extragalactic Radio Sources, Proceedings of a workshop held at Ringberg Castle, Tegernsee, FRG, September 22-28, 1991, ed. H.-J. Röser, \& K. Meisenheimer (Springer-Verlag, Berlin, Heidelberg, New York), also Lect. Notes Phys. 1993, 421, 243

Clarke, D. A., Norman, M. L., \& Burns, J. O. 1986, ApJ, 311, L63

Conway, J. E. 2002, New Astron. Rev., 46, 263

Curtis, H. D. 1918, Publications of Lick Observatory, 13, 11

Ferrari, A. 1998, ARA\&A, 36, 539

Heinz, S., Reynolds, C. S., \& Begelman, M. C. 1998, ApJ, 501, 126

Jones, T. W., Ryu, D., \& Engel, A. 1999, ApJ, 512, 105

Komissarov, S. S. 1999, MNRAS, 308, 1069

Kössl, D., \& Müller, E. 1988, A\&A, 206, 204

Krause, M. 2002, A\&A, 386, L1

Krause, M., \& Camenzind, M. 2001, A\&A, 380, 789

Krichbaum, T. P., Alef, W., Witzel, A., et al. 1998, A\&A, 329, 873

Lind, K. R., Payne, D. G., Meier, D. L., \& Blandford, R. D. 1989, ApJ, 344, 89

Loken, C., Burns, J. O., Clarke, D. A., \& Norman, M. L. 1992, ApJ, 392,54
Massaglia, S., Bodo, G., \& Ferrari, A. 1996, A\&A, 307, 997

Norman, M. L. 1993, in Space Telescope Science Institute Symposium Series, Proceedings of the Astrophysical Jets Meeting, held in Baltimore 1992 May 12-14 (Cambridge, UK: Cambridge University Press), ed. D. Burgarella, M. Livio, \& C. P. O'Dea, 211

Norman, M. L., Winkler, K. H. A., \& Smarr, L. 1983, in Astrophysical jets; Proceedings of the International Workshop, Turin, Italy, October 7-9, 1982 (A84-22076 08-90) (Dordrecht, D. Reidel Publishing Co.), 227-250, Discussion, p. 251

Norman, M. L., Winkler, K.-H. A., Smarr, L., \& Smith, M. D. 1982, A\&A, 113, 285

Nulsen, P. E. J., David, L. P., McNamara, B. R. et al. 2002, ApJ, 568, 163

Parma, P., Murgia, M., Morganti, R., et al. 1999, A\&A, 344, 7

Reynolds, C. S., Heinz, S., \& Begelman, M. C. 2002, MNRAS, 332, 271

Saikia, D. J., Thomasson, P., Spencer, R. E., et al. 2002, A\&A, 391, 149

Saxton, C. J., Sutherland, R. S., Bicknell, G. V., Blanchet, G. F., \& Wagner, S. J. 2002, A\&A, 393, 765

Scheck, L., Aloy, M. A., Martí, J. M., Gómez, J. L., \& Müller, E. 2002, MNRAS, 331, 615

Scheuer, P. A. G. 1995, MNRAS, 277, 331

Schoenmakers, A. P., de Bruyn, A. G., Röttgering, H. J. A., \& van der Laan, H. 2001, A\&A, 374, 861

Sedov, L. I. 1959, Similarity and Dimensional Methods in Mechanics (New York: Academic Press)

Smith, D. A., Wilson, A. S., Arnaud, K. A., Terashima, Y., \& Young, A. J. 2002, ApJ, 565

Snellen, I., \& Schilizzi, R. 2002, New Astron. Rev., 46, 61

Soker, N., Blanton, E. L., \& Sarazin, C. L. 2002, ApJ, 573, 533

Tregillis, I. L., Jones, T. W., \& Ryu, D. 2001, ApJ, 557, 475

Ziegler, U., \& Yorke, H. W. 1997, Comput. Phys. Commun., 101, 54 